\title{
REVIEW
}

\section{Voyage of RepA protein from plasmid DNA replication through amyloid aggregation towards synthetic biology}

\author{
Rafael Giraldo, María Elena Fernández-Tresguerres \\ Department of Chemical and Physical Biology, Centro de Investigaciones Biológicas (CIB) - CSIC, Madrid, \\ Spain
}

Received $1^{\text {st }}$ March 2010.

Revised $12^{\text {th }}$ April 2010.

Published online $12^{\text {th }}$ May 2010.

\begin{abstract}
Summary
DNA replication of plasmids in Gram-negative bacteria has been an object of study at CIB-CSIC for nearly 30 years. We have been focused on the enterobacterial antibiotic resistance factor R1 (1981-1992) and the pPS10 replicon from the phytopathogen Pseudomonas savastanoi (since 1984). Our group has used multidisciplinary (genetic, biochemical and biophysical-structural) approaches to unravel the molecular mechanism for the activation of RepA. Rep-type plasmidic proteins are either transcriptional repressors or replication initiators/inhibitors, depending on their association state (dimers vs. monomers) and targeting of alternative (operator or iteron) DNA sites. We discovered that allosteric DNA-binding remodels the structure of RepA N-terminal domain (WH1), transforming $\alpha$-helical portions into $\beta$-strands. This precisely tunes the distances between the DNA reading heads in WH1 and the C-terminal domain (WH2), to match the target operator or iteron sequences. We have recently moved into engineering such structural transformation in RepA-WH1 to build-up synthetic protein devices that allow for customized ligand (DNA)-promoted amyloidogenesis. Our basic studies on plasmid DNA replication are relevant for settling the bases of a minimalist bacterial model to tackle transmissible amyloid proteinopathies and are a valuable tool for bottom-up synthetic biology.
\end{abstract}

Key words: plasmid replication; protein amyloids; protein-DNA interactions; RepA protein; synthetic biology

\section{INTRODUCTION}

In the course of the more than 50 years of its existence, the Centro de Investigaciones Biológicas (CIB) of the Spanish National Research Council (CSIC) has played a key role in the establishment and

Rafael Giraldo, Centro de Investigaciones Biológicas (CIB) - CSIC, Ramiro de Maeztu 9, E-28040 Madrid, Spain

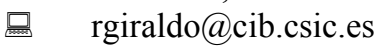

욜 +34-91-8373112

且 +34-91-5360432 success of Molecular and Cellular Biology in Spain, and, despite the challenges of the scientific, social, economic and even the political environment, has emerged in the forefront of research on microorganisms. With roots deep in bacterial and bacteriophage physiology, molecular genetics and biochemistry and, more recently, structural biology, current research on microbes at CIB is firmly entering its second half century aiming to tackle novel fundamental questions, together with biomedical, environmental and biotechnological challenges. With the aid of powerful omic tools, CIB is now moving towards contributing to a comprehensive understanding of life (systems biology) and engineering new biological functionalities and entities (synthetic biology). 
Plasmids are extrachromosomal DNA molecules that replicate autonomously in a host cell. They are ubiquitous in bacteria and very common in yeast, carrying gene cassettes responsible for expanding the metabolic abilities, and thus the evolutionary fitness, of their hosting population. At first sight, the plasmid biology field might nowadays seem a quiet, shallow scientific pond, eons apart from the raging waters of research on cancer, neurodegenerative diseases or ageing. However, leaving aside key contributions to the development of genetic engineering and molecular biology, plasmids continue to provide invaluable biological model systems and tools, not to mention their leading role as natural vectors for horizontal gene transfer across microbial populations, with both environmental and clinical implications. This short review article intends to lead the reader through three decades of research at CIB on two RepA proteins (those encoded by the distantly related R1 and pPS10 plasmids), from their seemingly modest origins as proteins required for plasmid DNA replication in Gram-negative bacteria, to the dramatic irruption of one of them (pPS10 RepA) as a paradigm for DNA-promoted allosteric conformational transitions in proteins, leading to a reliable model system to study and engineer protein amyloidosis. For extensive reviews on plasmid replication in bacteria, reading the references by del Solar et al. (1998), Krüger et al. (2004) and Paulsson and Chattoraj (2006) is strongly recommended. Gloria del Solar and Manuel Espinosa our colleagues at CIB carry out their research on pMV158, a plasmid from Gram-positive bacteria that replicates through a mechanism (the rolling circle) fundamentally different from R1 and pPS10, and which will thus not be reviewed here.

\section{ORIGINS OF RESEARCH ON PLASMID BIOLOGY IN GRAM-NEGATIVE BACTERIA AT CIB}

In the 1960's, young scientists arrived at CIB after spending their postdoctoral training abroad, with fresh ideas on how research was carried out in centres of excellence in Europe and USA. After faceing many difficulties, they succeeded in gaining international scientific prestige. New Institutes and groups were created: Cellular Biology (1964) was led by Manuel Losada (studies on photosynthesis) and from 1969, when he moved to the University of Seville, by David Vázquez (mechanism of action of antibiotics); Enzymology, a discipline that had emerged in 1958 at the Institute Marañón, was led by Alberto Sols (carbohydrate metabolism), considered the founder of modern Biochemistry in Spain; Molecular Biology was introduced in 1967 by Eladio Viñuela and Margarita Salas, with their studies on the bacteriophage Ø29.

In the 1970's, due to lack of space, some of the aforementioned groups moved to constitute new research Institutes across the country, leaving CIB to establish a base for Molecular and Cell Biology in Spain. In parallel, the Institute of Cellular Biology underwent structural changes and departments and sections were created according to new emergent needs. Thus, a section on Enzymatic Regulation was established at the Department of Cellular Chemistry by José Luis Cánovas in 1969, once he was back after postdoctoral studies at the University of California at Berkeley (USA). He studied the regulation of biosynthetic and degradative pathways of carbohydrates and hydroaromatic compounds in Pseudomonas putida and Acinetobacter calcoaceticus. One of us (M.E.F.-T) got her PhD in 1971 working on that subject and then went for a postdoctoral at the University of Leicester (UK), where she studied the genetics and physiology of the integration of the F plasmid in Escherichia coli chromosome (Tresguerres et al. 1975). This was actually the first contact of any scientist from CIB with plasmid biology. When in 1975 J. L. Cánovas became the Director of the Institute of Cell Biology, the areas of interest in the Unit of Enzymatic Regulation were widened to include the control of cell division and growth in $E$. coli and of carbohydrate catabolism in P. putida. In 1976, Ramón Díaz-Orejas defended his PhD Thesis on the latter subject. In 1979, Cellular Biology was restructured into eight Structural Units of Research. The Unit of Physiology and Bacterial Genetics, led by Gertrudis de Torrontegui, continued the topics of study mentioned above, except the genetic control of carbohydrate degradation.

\section{PLASMID R1 REPLICATION: GETTING FAMILIAR WITH PROTEIN AGGREGATION}

In 1981, back from working at the labs of Kurt Nordström in Odense (Denmark) and Walter Staudenbauer in Berlin, Ramón Díaz-Orejas brought with him a pioneering cell-free, transcription-translation coupled, assay to replicate the enterobacterial plasmid R1 in vitro (Díaz and Ortega 1984, Díaz et al. 1981). After succeeding in establishing molecular biology tools in a lab 
previously devoted to bacterial physiology, he and his first PhD student, Sagrario Ortega, found that R1 plasmid could be made to replicate in ammonium sulphate-fractionated $E$. coli extracts supplied with the purified, albeit aggregated as inclusion bodies (IBs), RepA replication initiator protein (Ortega et al. 1986). They were also able to dissect the requirement for a number of host replication proteins, either using extracts from thermosensitive mutants of those factors or depleting them with polyclonal antibodies. Actually, their results provided an early example of a protein retaining its activity even in an aggregated state: probably molecular chaperones present in the cell extract were solubilizing a fraction of the protein from the IBs, as was demonstrated shortly afterwards by scientists working with another Rep-type protein (Wickner et al. 1991). Ramón was the core and the leader of the group until 2003, when he left plasmid replication to become fully devoted to yet another one of his "scientific children": the toxin-antitoxin (TA) system parD which he, together with Alicia Bravo and Gertrudis de Torrontegui, had found in plasmid $\mathrm{R} 1$ (see in this issue the review on TA systems).

It was then evident that progress in the understanding of RepA binding to the replication origin of $\mathrm{R} 1$ (oriR) would require having it in a soluble form. This bottleneck was tackled by one of us (R.G.) in his PhD Thesis. After more than two years of unsuccessful assaults to protein solubilization in an active form, a dialysis procedure in the presence of the detergent CHAPS was devised, to shield aggregation-prone surfaces in the protein while removing the chaotropic agent used to solubilize RepA from the IBs, thus refolding the protein (Giraldo and Díaz 1992). The resulting protein preparations were 40-fold more active than aggregated RepA in the in vitro replication assay and opened the way to a full characterization of RepA-oriR binding, by means of electrophoretic mobility-shift assays (EMSAs) and DNaseI/hydroxyl-radical footprinting. These studies allowed us to reveal the presence of two imperfect palindromic (inverted) DNA repeats, in-phase at the edges of origin DNA: the first of them was bound with high affinity by RepA, then another protein molecule was bound to the distal, lower affinity site, resulting in DNA looping and subsequent filling of the intervening DNA sequence driven by cooperative protein-protein interactions (Giraldo and Díaz 1992). This model stands as a paradigm for the assembly of a nucleoprotein complex at a replication origin devoid of directly repeated DNA sequences. In addition, the detergent-assisted refolding procedure has since then been successfully used with many different aggregated recombinant proteins, both in CIB and in other locations. Finally, experiments were carried out to study the RepA-driven binding of the chromosomal initiator protein DnaA, an essential factor in R1 replication, to oriR (Ortega-Jiménez et al. 1992) and, in collaboration with Masamichi Kohiyama's group in Paris, the requirement of the DnaK chaperone for an efficient replication of this plasmid (Giraldo-Suárez et al. 1993).

Although all these were valuable advances in understanding plasmid replication in Gram-negative bacteria, R1 RepA protein was still difficult to handle at high concentrations and thus we looked for a more soluble and, if possible, smaller Rep protein. This would open a way to carry out the biophysical and structural studies that, in those days (early 1990's), were mandatory for further progress in unravelling the mechanism of plasmid replication.

\section{pPS10 RepA: A VERSATILE DNA BINDING PROTEIN}

In 1984, Concha Nieto started her PhD Thesis on a new plasmid replicon (pPS10) that she isolated from Pseudomonas savastanoi, a pathogen of the olive tree. Besides Ramón Díaz-Orejas, she was also supervised by one of us (M.E.F.-T.), who has since been linked to the study of this plasmid replicon. An extensive review on our research on pPS10 and its replication protein (RepA) has been published elsewhere (Giraldo and Fernández-Tresguerres 2004).

pPS10 minimal replicon was initially isolated with the intention of providing a source of specific vectors for genetic manipulation of Pseudomonadaceae (Nieto et al. 1990). However, far beyond such a purpose, DNA sequencing revealed a combination of both a minimal origin of replication (oriV) and a relatively short, and thus presumably structurally simple, replication protein (RepA) (Nieto et al. 1992) (Fig. 1 A). Our first studies on the molecular mechanism of pPS10 replication were focused on the determinants of the dual functions of RepA as a transcriptional auto-repressor and as an initiator of plasmid DNA replication. Such issues were at that time hotspots in research on plasmid replication (del Solar et al. 1998, Krüger et al. 2004, and references therein). We characterized the differential binding of RepA dimers to an inversely repeated 8 bp operator sequence overlapping with the promoter of the rep $A$ gene (García de Viedma et al. 1995a), and of RepA monomers to the four identical $22 \mathrm{bp}$ iteron repeats (García de Viedma et al. 1996) that, together with a binding site for the chromosomal initiator DnaA (Nieto et al. 1992), constitute the nucleoprotein 
complex that initiates DNA replication. Pioneering analytical ultracentrifugation studies, in collaboration with our colleague at CIB Germán Rivas, determined that dimers were the most abundant RepA species in solution (García de Viedma et al. 1996).

Studies to determine the molecular basis for the narrow host-range of pPS10 replication in vivo were begun under the leadership of M.E.F.-T. We first succeeded in making the plasmid replicate in cell-free extracts from $E$. coli carrying a shuttle vector expressing RepA, thus determining the requirement for a number of host factors in DNA replication (Giraldo et al. 1992). Then, chemical mutagenesis of the plasmid allowed for the isolation of point mutations in repA that expanded the host range of the plasmid to Enterobacteria (Fernández-Tresguerres et al. 1995, Maestro et al. 2003). These experiments showed that the most common broad host-range mutation resulted in the change of Ala31 to Val. Such a mutation would show later on to have fascinating pleiotropic effects. Subsequent random mutagenesis of the E. coli chromosome, in collaboration with Martine Coutourier's group in Belgium, led to the isolation of a triple mutant in the dnaA gene that efficiently supported the establishment of wild-type pPS10 (Maestro et al. 2002).

Another venue in pPS10 RepA research was the quest for sequence motifs in RepA that could be involved in protein-protein and protein-DNA interactions (Giraldo et al. 1989, García de Viedma et al. 1995b). However, advancing our knowledge on the molecular basis for the apparently antagonistic functions of RepA was waiting for structural studies that, in those days, were beyond our skills.

\section{DNA-PROMOTED CONFORMATIONAL ACTIVATION OF RepA}

In 1995, one of us (R.G.) returned from a postdoctoral stay on structural biology and biophysics at the MRC Laboratory of Molecular Biology in Cambridge (UK), and started a new phase in RepA research (Fig. 1 B). We first mapped the existence of two domains in RepA that, based on their potential secondary structures, were correctly predicted to have Winged-Helix (WH) folds. The N-terminal domain (WH1) was found to be responsible for RepA dimerization, whereas the C-terminal domain (WH2) was binding to the $6 \mathrm{bp}$ sequence common to the operator half repeats and to the core of each iteron. Interestingly, a deletion mutant with enhanced monomerization showed that, in the monomers, WH1 has an ancillary role to $\mathrm{WH} 2$ in binding to iteron
DNA (Giraldo et al. 1998). Hydrodynamic studies on RepA binding to an iteron DNA sequence showed that this was sufficient to displace the equilibrium towards protein monomerization, coupled to a conformational change in WH1 that, according to circular dichroism spectroscopy, increased its contents in $\beta$-sheet at the expense of $\alpha$-helices (Díaz-López et al. 2003). These results were later extended by means of fluorescence spectroscopy studies, in collaboration with Pilar Lillo's group at the CSIC Institute of Physical-Chemistry, defining sequential steps in the recognition of an iteron by a RepA dimer, and its subsequent dissociation into monomers. Such low affinity RepA-iteron DNA binding was enhanced by macromolecular crowding conditions intended to mimic in vitro the intracellular milieu (Díaz-López et al. 2006, Diederix et al. 2008).

A breakthrough in our understanding of Rep-type proteins came in 1999 from RepE, the DNA replication initiator of $\mathrm{F}$ plasmid, which had been studied for a long time by Chieko Wada in Japan. Her group, in collaboration with Kunio Miki, solved the structure of the monomeric species RepE54 bound to an iteron DNA oligonucleotide (Komori et al. 1999). This confirmed most of our previous work on RepA domains and DNA binding and allowed us to model by homology the structure of a RepA monomer. Meanwhile, we had been working, together with our colleague at CIB Antonio Romero, in the crystallization of RepA-WH1 dimers, the most soluble protein construct we managed to handle. We succeeded in solving its structure to $2.75 \AA$ of resolution (Giraldo et al. 2003). Comparison of the structure of a RepA-WH1 dimer with the model for its monomeric form, revealed the molecular mechanism for the activation of Rep-type proteins (for details, Fig. 1 B). In summary, the RepA-WH1 dimer exhibits an $\alpha+\beta$ fold in which a V-shaped bundle of three $\alpha$-helices is remodelled upon monomerization, releasing the C-terminal $\alpha$-helix which, together with the flexible $\mathrm{N}$-terminus of the domain, assemble into a short antiparallel $\beta$-sheet. In doing so, the WH1 and $\mathrm{WH} 2$ domains are brought closer together on the DNA in such a way that each one of them is able to recognize a halve of an iteron. This overall mechanism was confirmed later on by the crystal structure of a RepE dimer bound to the repE operator sequence (Nakamura et al. 2007).

Our final effort on pPS10 replication was trying to characterize handcuffing: the RepA-mediated protein bridges that pair together already replicated plasmid copies to inhibit premature re-initiation (for an excellent review on this issue, see Paulsson and Chattoraj 2006). We combined genetic selection of mutants affected in incompatibility with chemical 


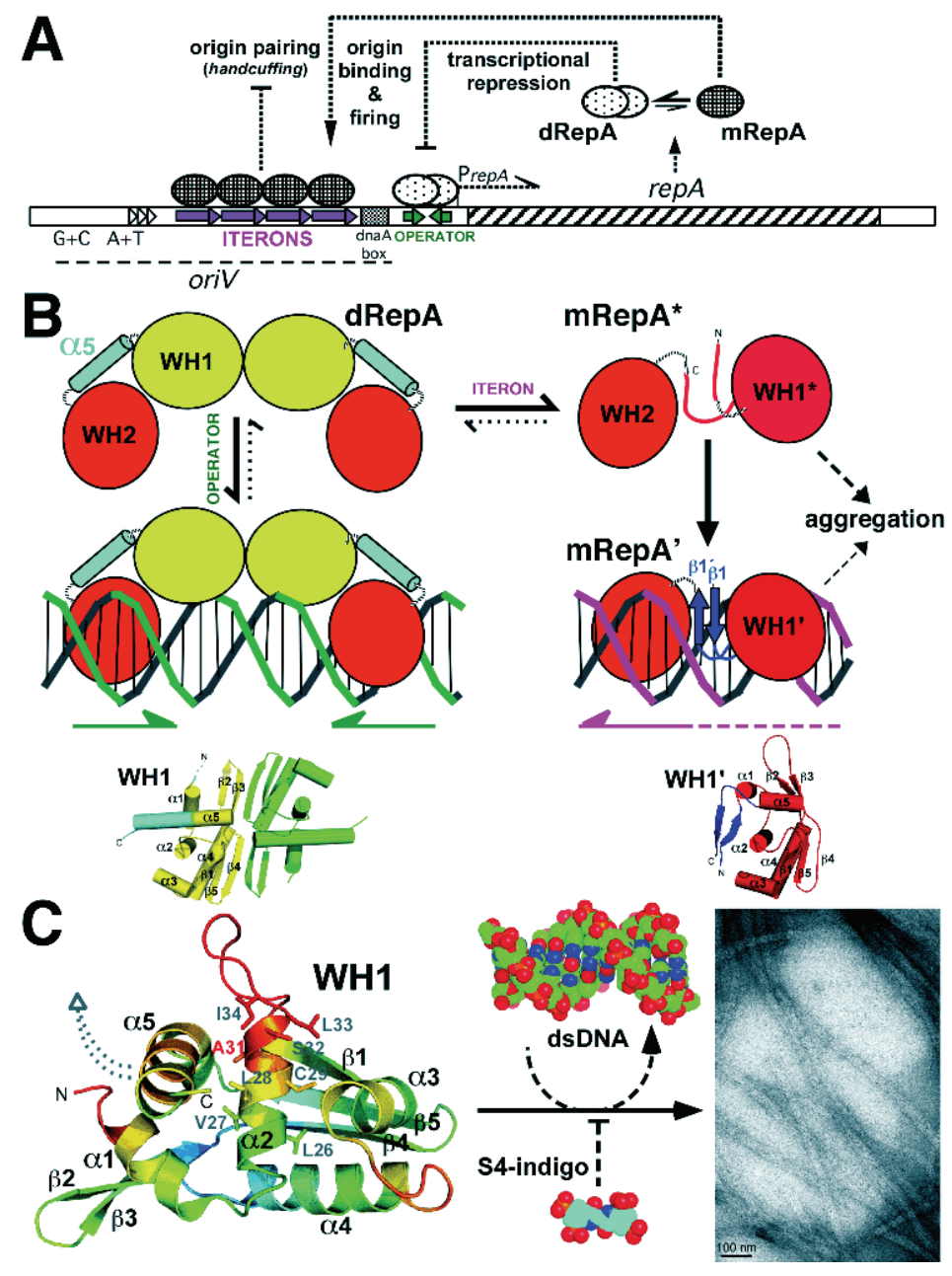

Fig. 1. RepA protein as a swiss army knife-like manifold device. A) The basic replicon of the P. savastanoi pPS10 plasmid. RepA 230 amino acid residue protein, binds as a dimer (dRepA) to an inversely-repeated DNA operator sequence (in green) to repress its own transcription. At the equilibrium, a minor RepA monomeric species (mRepA) binds cooperatively to four identical DNA tandem repeats (iterons, in magenta) at the replication origin (oriV) to initiate DNA replication. Once the origin region is replicated, iteron-bound RepA molecules bring together two plasmid molecules to inhibit premature origin re-firing. This cartoon model was the state of the art until 1997. B) In 1998 it was shown that RepA is actually composed of two Winged-Helix (WH) domains, being the N-terminal one (WH1) responsible for protein dimerization. Studies reported between 2003-06 showed that, on binding to operator DNA, the C-terminal WH2 domains in dRepA bind to each half of the inverted DNA repeat through the major grooves of the double helix, whereas WH1 binds to the phosphodiester backbone. Binding to each iteron DNA site actively dissociates dRepA into a short-lived metastable intermediate (mRepA*), which becomes stable (mRepA') upon WH1* binding to DNA. The (mRepA'-iteron) ${ }_{4}$ complex triggers conformational changes in origin DNA and the accretion of host factors to initiate replication. Monomeric species of RepA, in particular unbound mRepA*, are aggregation prone. In mRepA', this is relevant for coupling post-replicative origins (handcuffing). Crystallographic and modeling studies (bottom) revealed an extensive conformational remodelling upon monomerization affecting the $\alpha 1-\alpha 2-\alpha 5$ helix bundle in WH1: the C-terminal $2 / 3$ of $\alpha 5$ (cyan) are released from the $\alpha 1-\alpha 2$ latch and transformed, together with the N-terminal flexible extension, into a short antiparallel $\beta$-sheet ( $\beta 1-\beta 1$ ', blue), leaving the rest of the domain essentially unchanged. This alters the packing geometry between WH1 and WH2, enabling the differential reading of the distinctly spaced operator $v s$. iteron target half-sites on DNA. C) Since 2005 the amyloidogenic potential of DNA-induced conformational changes in RepA has been explored. A model of a dRepA-WH1 subunit (left), colour-coded according to crystallographic thermal B-factors (an indirect evidence for structural flexibility), shows that the C-terminal half of $\alpha 2$ and the following long loop have the potential to fluctuate. This overlaps with sequence stretch $\left(\mathrm{L}_{26} \mathrm{VLCA}_{-} \mathrm{SLI}_{34}\right)$ that, as a synthetic peptide and when carrying the broad host-range and high plasmid copy-number mutation A31V, assembles into an amyloid cross- $\beta$ structure. Transient binding to $11 \mathrm{bp}$ DNA oligonucleotides including the natural operator or iteron sequences results in local unfolding of WH1 (dotted arrow) and aggregation. The operator spacer sequence induces the formation of ordered amyloid fibres (right). A tetra-sulphonated derivative of the indigo stain competes with dsDNA for binding to WH1, thus inhibiting protein amyloidosis. Models were rendered with PyMOL (www.pymol.org). 
cross-linking, proteolysis, mass-spectrometry and electron microscopy (the latter in collaboration with Rudi Lurz in Berlin) to map residues involved in protein-protein interactions, which were then used as constrains to discriminate among various RepA-RepA docking models generated in silico (Gasset-Rosa et al. 2008a). We concluded that RepA monomers were directly involved in WH1-mediated pairing, but through a protein interface different to the dimerization $\beta$-sheet. Among the residues identified, it was Ala31, the same residue previously shown to broaden the host range of the plasmid when mutated to Val (Fernández-Tresguerres et al. 1995).

\section{UBIQUITOUS RepA-WH1 INITIATOR MODULES: AN INCURSION INTO EUKARYOTIC DNA REPLICATION}

At the turn of this new century very little was known on the structures of DNA replication initiators. Among the unknowns, the Origin Recognition Complex (ORC), responsible for DNA replication initiation in all the eukaryotes, was the Holy Grail (Bell 2002). With the wealth of knowledge on pPS10 RepA structure, although still very fragmentary, one of us (R.G.) undertook in 1998 a series of biochemical and spectroscopic studies on the fourth (Orc4p) of the six subunits of ORC from the yeast Saccharomyces cerevisiae. The starting evidence was very feeble: a low, residual sequence similarity between RepA-WH1 and the C-terminus of Orc4p, that included a few hydrophobic residues that we already knew were essential for RepA structure and function. Independently, a group led by James Berger in Berkeley reported the structure of the first archaeal ORC-like protein (Liu et al. 2000), which showed at its C-terminus a WH fold that perfectly matched our prediction for the yeast Orc $4 p$ initiator. The article containing these results (Giraldo and Díaz-Orejas, 2001) opened fascinating phylogenetic perspectives to the evolution of the DNA replication initiator machinery, in which plasmids and their Rep proteins could have had a role in the gene transfer of initiator genes from ancestral Bacteria to Archaea and Eukarya (Giraldo 2003, Grabowski and Kelman 2003, Giraldo and Fernández-Tresguerres 2004).

\section{ENGINEERING PROTEIN AMYLOIDOSIS IN $\operatorname{RepA}$}

RepA-WH1 has an astonishing ability to use distinct protein-protein interaction interfaces for different functional roles. In addition, in spite of its superior solubility when compared with R1 RepA, pPS10 RepA (and in fact all Rep-type proteins) still has a marked tendency towards aggregation upon DNA-promoted monomerization. Thus one of us (R.G.) decided to delve deeper into the basis for such an unpleasant (for a practicing biochemist) behaviour. We found (Fig. 1 C) that a region starting at the $\mathrm{C}$-terminus of helix $\alpha 2$ has the potential to aggregate as $\beta$-sheets: besides bioinformatics predictions in this sense, a synthetic peptide spanning such sequence readily assembled into an amyloid cross- $\beta$ structure. Amyloids are protein macromolecular assemblies formed by the stable accretion of short sequence stretches as strands in packed $\beta$-sheets of indefinite extension. The resulting fibres are common in many clinically relevant conformational proteinopathies, including Alzheimer's, Parkinson's, Huntington's and prion neurodegenerative diseases and some systemic amyloidoses (Chiti and Dobson 2006). We were able to take advantage of the DNA-promoted conformational transformation in RepA-WH1, combined with the pleiotropic mutant variant A31V (see above), to modulate the assembly of this protein into quite homogeneous nano-structures, such as spherulites and fibres (Giraldo 2007). In parallel, in a search for inhibitors of RepA binding to DNA, we found that tetra-sulphonated derivatives of indigo blue were able to compete, with reasonable affinity, with the specific pPS10 operator and iteron DNA sequences for binding to WH1. In doing so, they also inhibited RepA-WH1 amyloidosis (Gasset-Rosa et al. 2008b). The importance of these results is that they constitute a proof of concept for the central role of allosteric DNA binding in RepA-WH1 amyloidogenesis. It is also a pioneering example of inhibition of amyloidosis by targeting a protein at a site distinct to that directly participating in $\operatorname{cross}-\beta$ assembly. Nowadays, pPS10 RepA-WH1 has thus turned out to be a powerful tool in building-up synthetic macromolecular assemblies with potential nano-technological applications, and a useful model system to explore the molecular basis and the inhibition of protein amyloidosis.

\section{CONCLUDING REMARKS}

A well-established trend in modern biological sciences is an overwhelming pressure on scientists to work on problems and model systems with obvious clinical or applied (technological, environmental) interest. In this sense, being devoted for such a long time to the essentials of the biology of prokaryotic 
extrachromosomal genetic elements might have appeared to many to be a trivial pursuit. On the contrary, our research on Rep-type replication proteins has provided considerable insight into the molecular basis not just of plasmid DNA replication in bacteria, but also on their eukaryotic initiator counterparts, on allosteric conformational changes in proteins and on the principles of protein aggregation. In the latter sense, at the corner stone of our third decade of research on Rep proteins at CIB, we are now exploring the emergent venue of bottom-up synthetic biology: their potentials as protein manifolds in engineering macromolecular devices with novel functionalities, both in vitro and in vivo.

\section{ACKNOWLEDGEMENTS}

Our research is currently supported by grants from the Spanish Ministry of Science and Innovation (MICINN: BIO2009-06952 and CSD2009-00088) and the Autonomous Government of Madrid (CAM: P-BIO-0214-2006). We are grateful to all the past and present members of our group, in special to our friends Ramón Díaz-Orejas and Ana Serrano, for their input and dedication to pPS10 and RepA for so many years.

\section{REFERENCES}

Bell SP: The origin recognition complex: from simple origins to complex functions. Genes Dev 16:659-672, 2002.

Chiti F, Dobson CM: Protein misfolding, functional amyloid, and human disease. Annu Rev Biochem 75:333-366, 2006.

del Solar G, Giraldo R, Ruíz-Echevarría MJ, Espinosa M, Díaz R: Replication and control of circular bacterial plasmids. Microbiol Mol Biol Rev 62:434-464, 1998.

Díaz R, Ortega S: Initiation of plasmid R1 replication in vitro is dependent of transcription by host RNA polymerase. Nucleic Acids Res 12:5175-5191, 1984.

Díaz R, Nordström K, Staudenbauer WL: Plasmid R1 DNA replication dependent on protein synthesis in cell-free extracts of $E$. coli. Nature 289:326-328, 1981.

Díaz-López T, Lages-Gonzalo M, Serrano-López A, Alfonso C, Rivas G, Díaz-Orejas R, Giraldo R: Structural changes in RepA, a plasmid replication initiator, upon binding to origin DNA. J Biol Chem 278:18606-18616, 2003.
Díaz-López T, Dávila-Fajardo C, Blaesing F, Lillo MP, Giraldo R: Early events in the binding of the pPS10 replication protein RepA to single iteron and operator DNA sequences. J Mol Biol 364:909-920, 2006.

Diederix REM, Dávila-Fajardo C, Giraldo R, Lillo MP: Fluorescence studies of the replication initiator protein RepA in complex with operator and iteron sequences and free in solution. FEBS J 275:5393-5407, 2008.

Fernández-Tresguerres ME, Martín M, García de Viedma D, Giraldo R, Díaz-Orejas R: Host growth temperature and a conservative amino acid substitution in the replication protein of pPS10 plasmid influence plasmid host range. J Bacteriol 177:4377-4384, 1995.

García de Viedma D, Giraldo R, Ruíz-Echevarría MJ, Lurz R, Díaz-Orejas R: Transcription of repA, the gene of the initiation protein of the Pseudomonas plasmid pPS10, is autorregulated by interactions of the RepA protein at a symmetrical operator. $\mathrm{J}$ Mol Biol 247:211-223, 1995a.

García de Viedma D, Serrano-López A, Díaz-Orejas $\mathrm{R}$ : Specific binding of the replication protein of plasmid pPS10 to direct and inverted repeats is mediated by an HTH motif. Nucleic Acids Res 23:5048-5054, 1995b.

García de Viedma D, Giraldo R, Rivas G, Fernández-Tresguerres E, Díaz-Orejas R: A Leucine-Zipper motif determines different functions in a DNA replication protein. EMBO J 15:925-934, 1996.

Gasset-Rosa F, Díaz-López T, Lurz R, Prieto A, Fernández-Tresguerres ME, Giraldo R: Negative regulation of pPS10 plasmid replication: Origin pairing by zipping-up DNA-bound RepA monomers. Mol Microbiol 68:560-572, 2008a.

Gasset-Rosa F, Maté MJ, Dávila-Fajardo C, Bravo J, Giraldo R: Binding of sulphonated indigo derivatives to RepA-WH1 inhibits DNA-induced protein amyloidogenesis. Nucleic Acids Res 36:2249-2256, 2008b.

Giraldo R: Common domains in the initiators of DNA replication in Bacteria, Archaea and Eukarya: Combined structural, functional and phylogenetic perspectives. FEMS Microbiol Rev 26:533-554, 2003.

Giraldo R: Defined DNA sequences promote de assembly of a bacterial protein into distinct amyloid nanostructures. Proc Natl Acad Sci USA 104:17388-17393, 2007.

Giraldo R, Díaz R: Differential binding of wild-type and a mutant RepA protein to oriR sequence suggests a model for the initiation of plasmid R1 replication. J Mol Biol 228:787-802, 1992. 
Giraldo R, Díaz-Orejas R: Similarities between the DNA replication initiators of Gram-negative bacteria plasmids (RepA) and eukaryotes (Orc4p)/archaea (Cdc6p). Proc Natl Acad Sci USA 98:4938-4943, 2001.

Giraldo R, Fernández-Tresguerres ME: 20 years of the pPS10 replicon: Insights on the molecular mechanism for the activation of DNA replication in iteron-containing bacterial plasmids. Plasmid 52:69-83, 2004.

Giraldo R, Nieto C, Fernández-Tresguerres ME, Díaz R: Bacterial zipper. Nature 342:866, 1989.

Giraldo R, Martín M, Fernández-Tresguerres ME, Nieto C, Díaz R: Mutations within the minimal replicon of plasmid pPS10 increase its host range. In Hughes P, Fanning E, Kohiyama M (eds.): DNA Replication: The Regulatory Mechanisms, Springer Verlag, Berlin, 1992, pp. 225-237.

Giraldo-Suárez R, Fernández-Tresguerres ME, Díaz-Orejas R, Malki A, Kohiyama M: The heat-shock DnaK protein is required for plasmid $\mathrm{R} 1$ replication and it is dispensable for plasmid ColE1 replication. Nucleic Acids Res 21:5495-5499, 1993.

Giraldo R, Andreu JM, Díaz-Orejas R: Protein domains and conformational changes in the activation of RepA, a DNA replication initiator. EMBO J 17:4511-4526, 1998.

Giraldo R, Fernández-Tornero C, Evans PR, Díaz-Orejas R, Romero A: A conformational switch between transcriptional repression and replication initiation in the RepA dimerization domain. Nat Struct Biol 10:565-571, 2003.

Grabowski B, Kelman Z: Archaeal DNA replication: Eukaryal proteins in a bacterial context. Annu Rev Microbiol 57:467-485, 2003.

Komori H, Matsunaga F, Higuchi Y, Ishiai M, Wada C, Miki K: Crystal structure of a prokaryotic replication initiator protein bound to DNA at $2.6 \AA$ resolution. EMBO J 18:4597-4607, 1999.

Krüger R, Rakowski SA, Filutowicz M: Participating elements in the replication of iteron containing plasmids. In Funnell BE, Phillips GJ (eds.): Plasmid Biology, ASM Press, Washington DC, 2004, pp 25-45.

Liu J, Smith CL, DeRyckere D, DeAngelis K, Martin GS, Berger JM: Structure and function of Cdc6/Cdc18: Implications for origin recognition and checkpoint control. Mol Cell 6:637-648, 2000 .
Maestro B, Sanz JM, Faelen M, Couturier M, Díaz-Orejas R, Fernández-Tresguerres ME: Modulation of pPS10 host range by DnaA. Mol Microbiol 46:223-234, 2002.

Maestro B, Sanz JM, Díaz-Orejas R, Fernández-Tresguerres E: Modulation of pPS10 host range by plasmid-encoded RepA initiator protein. J Bacteriol 185:1367-1375, 2003.

Nakamura A, Wada C, Miki K: Structural basis for regulation of bifunctional roles in replication initiator protein. Proc Natl Acad Sci USA 104:18484-18489, 2007.

Nieto C, Fernández-Tresguerres E, Sánchez N, Vicente M, Díaz R: Cloning vectors, derived from a naturally occurring plasmid of Pseudomonas savastanoi, specifically tailored for genetic manipulations in Pseudomonas. Gene 87:145-149, 1990.

Nieto C, Giraldo R, Fernández-Tresguerres E, Díaz $\mathrm{R}$ : Genetic and functional analysis of the basic replicon of pPS10, a plasmid specific of Pseudomonas isolated from Pseudomonas syringae pv. savastanoi. J Mol Biol 223:415-426, 1992.

Ortega S, Lanka E, Díaz R: The involvement of host replication proteins and of specific origin sequences in the in vitro replication of miniplasmid R1 DNA. Nucleic Acids Res 14:428-435, 1986.

Ortega-Jiménez S, Giraldo-Suárez R, Fernández-Tresguerres ME, Berzal-Herranz A, Díaz-Orejas R: DnaA-dependent replication of plasmid R1 occurs in the presence of point mutations that disrupt the dnaA box of oriR. Nucleic Acids Res 20:2547-2551, 1992.

Paulsson J, Chattoraj DK: Origin inactivation in bacterial DNA replication control. Mol Microbiol 61:9-15, 2006.

Tresguerres EF, Nandadasa HG, Pritchard RH: Suppression of initiation negative strains of Escherichia coli by integration of the sex factor F. J Bacteriol 121:554-561, 1975.

Wickner S, Hoskins J, McKenney K: Function of DnaJ and DnaK chaperones in origin-specific DNA binding by RepA. Nature 350:165-167, 1991. 